\title{
The effects of cognitive style and emotional trade-off difficulty on information processing in decision-making
}

\author{
Dawei Wang ${ }^{1}$, Leilei Hao' ${ }^{1}$, Phil Maguire ${ }^{2}$, and Yixin $\mathrm{Hu}^{1}$ \\ ${ }^{1}$ School of Psychology, Shandong Normal University, Jinan, People's Republic of China \\ ${ }^{2}$ Department of Computer Science, National University of Ireland, Maynooth, Ireland
}

\begin{abstract}
7 his study investigated the effects of cognitive style and emotional trade-off difficulty (ETOD) on information processing in decision-making. Eighty undergraduates $(73.75 \%$ female, $M=21.90)$, grouped according to their cognitive style (field-dependent or field-independent), conducted an Information Display Board (IDB) task, through which search time, search depth and search pattern were measured. Participants' emotional states were assessed both before and after the IDB task. The results showed that participants experienced significantly more negative emotion under high ETOD compared to those under low ETOD. While both cognitive style and ETOD had significant effects on search time and search depth, only ETOD significantly influenced search pattern; individuals in both cognitive style groups tended to use attribute-based processing under high ETOD and to use alternative-based processing under low ETOD. There was also a significant interaction between cognitive style and ETOD for search time and search depth. We propose that these results are best accounted for by the coping behaviour framework under high ETOD, and by the negative emotion hypothesis under low ETOD.
\end{abstract}

Keywords: Cognitive style; Emotional trade-off difficulty; Information processing in decision-making.

Nobel Laureate Herbert Simon (1983) notably stated that his theory of bounded rationality would not be complete until the role of emotion in decision-making was specified. Since then, many researchers have further emphasised the view that a comprehensive theory of decision-making must take emotion into account (Lerner, Li, Valdesolo, \& Kassam, 2015). Although emotional factors have been excluded from rational decision theory, there can be little doubt that they play a significant role.

Currently, two themes, both supported by empirical findings, highlight the impact of emotion on decision-making. On the one hand, task-irrelevant emotion has been suggested to be influential. For instance, Bandyopadhyay, Srinivasan, and Pammi (2013) found that task-irrelevant emotional information significantly affected the process of risky decision-making, as well as participants' emotional experience. However, task-relevant (or task-induced) emotion has also been shown to affect decision-making. Palmer and Koenig-Lewis (2014) found that negative anticipatory emotion (i.e., a kind of task-induced emotion reflecting the affective response to a current stimulus) significantly influenced consumers' satisfaction. Given the differences in how emotion can influence decision-making, multiple studies have highlighted the importance of distinguishing task-induced emotion from task-irrelevant emotion (Baumgartner, Pieters, \& Bagozzi, 2008; Lerner et al., 2015; Li, Huang, \& Zhou, 2015).

Although task-induced emotion has become a prominent research target, the idea of using "trade-off difficulty" as a means of evoking such emotion during decision-making has not received as much attention. Emotional trade-off difficulty (ETOD) was firstly proposed by Payne, Bettman, and Eric (1993). According to their research, if a decision-maker engages in a mental process that involves some trade-off difficulty (e.g., decisions involving self-relevant targets such as life, health, environmental protection or time), negative emotions and mental conflicts build up relative to the difficulty of evaluating the trade-off. Several studies have investigated

Correspondence should be addressed to Yixin Hu, School of Psychology, Shandong Normal University, No. 88, East Wenhua Road, Jinan, Shandong Province 250014, People's Republic of China. (E-mail: huyixin2005@163.com).

This study was supported by National Natural Science Fund of China (grant no. 31471002), Natural Science Foundation of Shandong Province of China (grant no. ZR2014CM033), Key R \& D program in Shandong Province of China (grant no. 2015GSF120015), Young People of Humanities and Social Sciences of the Ministry of Education of China (grant no. 15YJC190006), Key Construction Project of Applied Psychology of Shandong Normal University of China and "The 12th Five" Emphasis Subject of Development and Education Psychology of Shandong Province of China. 
how decision-making is affected by task-induced negative emotion generated during the evaluation process. These studies confirm that ETOD can influence the decision made, insofar as individuals processing high ETOD tend to postpone dealing with the problem in order to reduce the associated negative emotion (Luce, Bettman, \& Payne, 2001; Zhou, 2010). Peng (2008) also found that consumers' decision-making behaviour showed a significant preference reversal in a matching-choice task under high ETOD.

A limitation of these studies is that they only explore the effect of ETOD at the level of decision-making outcomes. More recent judgement and decision-making research considers not only the final outcome but, more constructively, seeks to reveal the "black box" through which mental processing delivers these outcomes. Indeed, an important feature of studies pertinent to ETOD is that negative emotion generated during the processes of trade-off is treated as an independent variable, and used to investigate whether task-induced emotion affects information processing in decision-making ( $\mathrm{Li}, \& \mathrm{Fu}, 2004)$.

\section{Theoretical explanations of information processing}

To date, studies investigating the effect of ETOD on information processing have mainly concentrated on task-induced negative emotion. The negative emotion hypothesis, which is based on both the effort-accuracy framework (Payne et al., 1993) and the coping behaviour framework (Susan \& Lazarus, 1988), has been applied to account for the effect of negative emotion induced by trade-off difficulty during decision-making.

The negative emotion hypothesis consists of two diametrically opposed views. One view is that negative emotion distracts an individual's attention and reduces the level of cognitive resources allocated to the processing of the task. This dip leads individuals to rely on heuristic strategies and attribute-based processing (Lewinsohn \& Mano, 1993). The other view is that the negative emotion subconsciously encourages or compels individuals to maximise their understanding of the decision-making environment, with an emphasis on information accuracy. This makes individuals more likely to make use of analytical, extensive strategies and alternative-based processing (Lazarus, 1991).

Susan and Lazarus (1988) proposed that this conflict can be interpreted in terms of coping behaviour. Specifically, they argued that evoked emotion leads to two coping styles: problem-focused coping and emotion-focused coping. In some cases, individuals may be motivated to engage in problem-focused coping, approaching emotion-laden decisions with the aim of resolving them to the best of their abilities. This idea is consistent with the observation that decision-makers shift towards more effortful, accurate processing when dealing with an emotional situation. However, emotion-focused coping refers to the idea that negative emotion may also precipitate avoidance, with individuals viewing it as a "burden" to be alleviated quickly. This attitude may lead to more cursory decision processing, and the use of simple, heuristic decision strategies. This emotion-focused coping can involve elements of both avoidance and altering the subjective meaning of a situation. In sum, these two conflicting coping styles can bias the meaning of the same experimental situation in markedly different ways. Furthermore, the two styles, or problem-viewing attitudes, may occur simultaneously in the same individual for a range of situations evoking negative emotions.

Several studies have suggested that, under high level ETOD, individuals tend to use attribute-based processing, with increased search expenditure and information acquisition (Luce, 1998; Luce, Bettman, \& Payne, 1997). Zhou (2010) also found that higher ETOD increased the time participants spent searching for information, and that individuals in such conditions tended to use attribute-based processing. These findings are not easily compatible either with the negative emotion hypothesis (based simply on the effort-accuracy framework), or with the coping behaviour framework. The observation that both effort expended and demand for accuracy (i.e., elongated information searching) increased simultaneously with the switch to attribute-based processing is something which both frameworks completely failed to predict.

One issue standing in the way of resolving this impasse is that previous studies have concentrated only on the specifics relevant to one of the frameworks, while neglecting to collect information relevant to the other side. Moreover, information on the participants themselves is commonly missing in such studies, making it difficult to evaluate the impact of individual mental processing. As stated by Damasio (1995), the effect of emotion on judgement and decision-making depends on individual differences, task characteristics and their interactions. In light of this, we believe that further investigation of individual differences may provide the key to reconciling the two classical frameworks, this being the focus of the current research.

\section{Cognitive style}

Cognitive style has long been considered an essential variable in addressing individual differences. For instance, Witkin (1964) proposed a one-dimensional model of variation in cognitive style, identifying the categories of field-dependent (FD) and field-independent (FI) for describing how people process information. Different abilities in mobilising and/or allocating mental-attentional capacity are considered to be the 
most likely explanation for differences in field-cognitive style. For example, FD individuals tend to collect extensive information from the environment, and therefore have difficulty in separating detailed incoming information from its contextual background. In contrast, FI individuals are more self-directed and task-focused; by relying on their internal experiences and logical analyses, they can more easily pick out the desired information. Research has shown that field-cognitive style can influence decision behaviour in different scenarios, including risky decision-making, crisis decision-making and managerial decision-making. For example, Chen (2011) found that individuals' cognitive styles played a significant role in determining how they responded to advice in group situations. FI individuals tended to stick to their initial judgement and were reluctant to adopt the recommendations of others, whereas FD individuals gave more careful consideration to others' views.

Recent studies have specifically addressed the effect of cognitive styles on information processing. Using eye tracking technology, Mawad, Trías, Giménez, Maiche, and Ares (2015) documented that FD consumers spent less time selecting a preferred yoghurt label and acquired less information than FI consumers engaged in the same task. Other studies have investigated the possibility of an interaction between field-cognitive style and emotion in decision-making. For example, Luo (2011) found that field-cognitive style can interact with emotion to influence people's impulsive buying behaviour. FD participants with positive emotion, but not negative emotion, were more likely to purchase products impulsively, whereas FI participants did not demonstrate a significant difference. In contrast, Qiu and $\mathrm{Wu}$ (2005) found that the interactive effect of field-cognitive style and negative emotion on travel decisions was not significant. These conflicting results highlight that the relationship between cognitive style and negative emotion poses an interesting question to be investigated.

\section{The present study}

In sum, previous studies have suggested that field-cognitive style may interact with emotion to influence decision-making behaviour. However, because those studies employed only task-irrelevant emotion, it remains unclear whether field-cognitive style interacts with task-induced emotion to influence information processing in decision-making. In the current study, we investigated how individuals with differences in field-cognitive style conducted their information search under different levels of ETOD. The primary aim of this study was to investigate how ETOD and field-cognitive style together influence search time, search depth and search pattern. According to the definitions provided by Payne (1976), search time refers to the average time in milliseconds that participants spend on all choice trials.
Search depth can then be calculated by subtracting the number of cells participants search by the total number of cells presented to them in each trial. Lastly, the search pattern can be calculated by subtracting the number of attribute-based transitions for a trial from the number of alternative-based transitions for that trial, and then dividing this difference by the sum of alternative-based and attribute-based transitions (ranged from -1 to 1 , with a higher value indicating attribute-based processing).

The investigation aimed to provide empirical evidence from both the situation and participant-based perspective, permitting an analysis of the underlying mechanisms of decision-making. In particular, the following hypotheses were investigated:

1 Individuals experience significantly more negative emotion under high ETOD than under low ETOD.

2 The "negative emotion hypothesis," which is based on the effort-accuracy framework, can be combined with the "coping behaviour framework" to provide a more comprehensive explanation of information processing in decision-making under the condition of ETOD.

3 Field-cognitive style interacts with ETOD to influence parameters relating to information processing including search time, search depth and search pattern.

\section{METHODS}

\section{Participants}

The Embedded Figures Test (EFT) was administered to two hundred undergraduates recruited from a Chinese university. Students were awarded course credit for participating; none had previously been involved in a similar experiment. A total of 80 undergraduates aged 19-25 years (59 female, 21 male, $M=21.90$ ) were chosen and divided into two groups according to their EFT scores. Forty students (30 females, 10 male, $M=20.70$ ) who scored $>18$ were categorised as the FI group, while another 40 (29 females, 11 male, $M=21.23$ ) who scored $<13.5$ were categorised as the FD group. An independent samples $t$-test revealed a significant difference in participants' EFT scores $(t(78)=19.59, p<.001)$.

All procedures performed in studies involving human participants were in accordance with the ethical standards of the research committee of Shandong Normal University, which is responsible for ethical review. Informed consent was obtained from all individual participants included in the study.

\section{Tasks and materials}

\section{Information Display Board}

The Information Display Board (IDB), developed by Payne (1976), aims at specifying participants' 
information acquisition patterns. The information in the IDB was hidden behind covered cells, with participants required to open one cell at each time by moving the mouse onto the cell in order to acquire useful information. Participants could scan the value hidden in the cell, but once the mouse was moved from the cell, the value was immediately and automatically hidden again. The computer program used to present the IDB automatically recorded the order in which the cells were opened, the time spent searching each cell and the final option chosen. These data were used to establish measures for illuminating the decision-making process, namely, search time, search depth and search pattern.

The development of ETOD materials and the specific operational method of the different levels of ETOD are presented in the Appendix.

\section{Embedded Figures Test}

The EFT is a timed paper-and-pencil performance test adapted from the individual-administered EFT. The present study employed a Chinese version of the EFT, as revised by Xie and Zhang (1988). The revised EFT retains the majority of the original EFT items, with a few complex figures slightly modified. It consists of three sections: section A includes 9 figures, while sections $\mathrm{B}$ and $\mathrm{C}$ include 10 figures. The test asks participants to locate and trace simple figures from complex figures within the three subsections as quickly as possible. The first section is used for practicing, and the total number of correct answers on the second and third sections (ranged between 0 and 20) is taken as the EFT score. These modifications to the original EFT were based on pilot testing with four groups (adults, senior-high-school students, junior-high-school students and primary-school students). The validity of the revised EFT has been examined using Pearson's correlation coefficients between the EFT and the rod-and-frame test (RFT) $(r=.49, p<.05)$. The test reliability has been assessed based on Pearson's correlation coefficients between section B and section C of the revised EFT $(r=.90, p<.05)$. The difficulty distribution of the revised EFT is .97-.21; the discrimination distribution is .17-.94.

\section{Positive and Negative Affect Scale}

The Positive and Negative Affect Scale (PANAS), developed by Watson, Clark, and Tellegen (1988), is one of the most widely used affect scales in psychology and other cognate disciplines. The PANAS measures two primary dimensions of mood-positive emotion (positive affect, PA) and negative emotion (negative affect, NA). Participants are required to rate the intensity of emotion experienced within a certain time. PA reflects their level of pleasurable engagement. In contrast, NA is a general measure of subjective distress. The two dimensions of affect (PA and NA) are not thought to be opposites. Zhang, Diao, and Constance (2004) found that the scale's two-dimensional structures have cross-cultural consistency. The Chinese version of the PANAS demonstrates high reliability, with a Cronbach's $\alpha=.87$, in line with the requirements of psychometrics.

\section{Procedure}

Participants in both the FI and FD group were further randomly divided into two groups, with each participant in the four groups assigned to a single experimental treatment. During the experiment, task guidance and the contents in the IDB were presented on a computer screen; participants performed the task individually. After providing informed consent and receiving an explanation of the experiment, they began the experimental procedure. Participants were required to enter basic personal information (including student number, age and gender), and were then presented with the next screen, where they were required to read the information carefully. First, they needed to complete the PANAS based on their current emotional experience. Then, once participants entered the exercise phase, they could practice one or more times depending on their own needs, without results being recorded.

After mastering the process, participants chose to enter the formal decision task, in which they needed to click on the information in the cell matrix to reveal options. The decision task involved four trials. The computer screen presented four house options, one by one, with five attributes in a table in each trial (see Table 1). All values were covered, and participants needed to click a box to view the hidden information. Participants viewed the information and made a choice. After completing the four trials, participants completed the PANAS again based on their emotional state. Finally, the participants were thanked for participating in the experiment.

SPSS19.0 was used for data storage and statistical analysis.

\section{RESULTS}

\section{Negative emotion measured by the PANAS}

Before participants completed the IDB, we used the PANAS to test the participants' negative emotion. We conducted a 2 (field-cognitive style: FI/FD) $\times 2$ (ETOD: high/low) $\times 2$ (gender: male/female) ANOVA for pre-test negative emotion. No main effects or interactions were significant (for all tests, $p>.05$ ); there was no significant difference in task-induced negative emotion among participants before beginning the experiment. 
TABLE 1

IDB task used for practicing

\begin{tabular}{lccccc}
\hline & \multicolumn{3}{c}{ High/Low ETOD } \\
\cline { 2 - 6 } & $\begin{array}{c}\text { Traffic condition } \\
\text { /Space interval }\end{array}$ & Security/Architectural style & House style/Landscape & Location/Ventilation & $\begin{array}{c}\text { Property management/ } \\
\text { Residential scale }\end{array}$ \\
\hline House 1 & Worse & Better & Average & Average & Best \\
House 2 & Average & Average & Best & Better & Worst \\
House 3 & Best & Worst & Worse & Average & Average \\
House 4 & Worst & Best & Average & Worst & Better \\
\hline
\end{tabular}

Note: All information under high or low ETOD is presented in a matrix format with rows representing alternatives and columns representing attribute. Each trial comprises different attribute values. We used five values to describe each attribute, namely average, better, best, worse and worst.

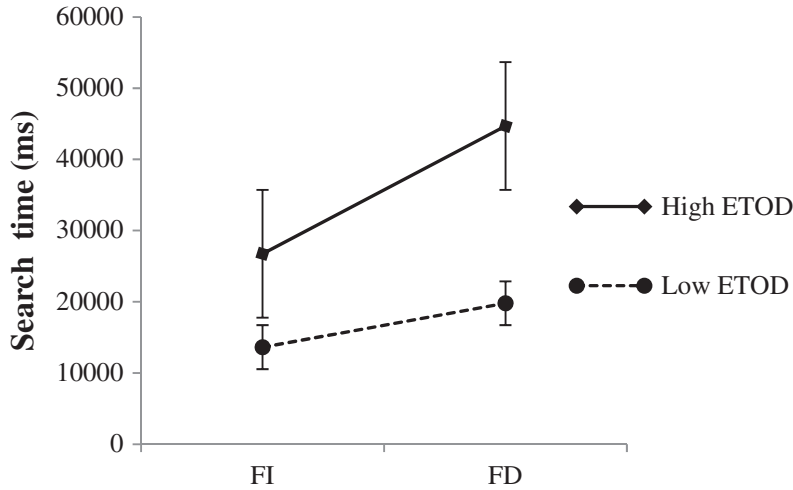

Figure 1. Interaction effect of field-cognitive style and ETOD on search time.

After participants completed the IDB task, we used the PANAS to retest their negative emotion. We conducted a 2 (field-cognitive style: FI/FD) $\times 2$ (ETOD: high/low) $\times 2$ (gender: male/female) ANOVA on post-test negative emotion, which revealed a significant main effect of ETOD on the level of negative emotion, $F(1$, 72) $=55.08, p<.001, \eta_{\mathrm{p}}^{2}=.43$. Participants under high ETOD scored significantly higher than those under low ETOD. No interaction or other main effects were significant (for all tests, $p>.05$ ), indicating that the variable of cognitive style did not stratify participants' experienced negative emotion under the same level of ETOD.

Examining task-induced negative emotion as an independent variable, we conducted a linear regression analysis on the three indicators of information processing. This analysis revealed that negative emotion significantly influenced search time, $\beta=.49, p<.001, R^{2}=.24$; search depth, $\beta=.54, p<.001, R^{2}=.29$ and search pattern, $\beta=-$ $.30, p<.01, R^{2}=.09$.

\section{Results of the IDB task}

Given that participants completed four trials in the formal experiment, we used the average score of the results in each experimental condition for statistical analysis.
TABLE 2

Search time $(\mathrm{ms})$ under high/low ETOD $(M \pm S D)$

\begin{tabular}{lcc}
\hline & High ETOD & Low ETOD \\
\hline FI & $26746.12 \pm 3968.26$ & $13630.14 \pm 2695.86$ \\
FD & $44691.13 \pm 12018.46$ & $19795.14 \pm 4051.70$ \\
\hline
\end{tabular}

Firstly, we conducted a 2 (field-cognitive style: FI/FD) $\times 2$ (ETOD: high/low) ANOVA on search time, which revealed two significant main effects (Table 2). One significant main effect was that FD participants searched longer than FI participants, $F(1,76)=63.23$, $p<.001, \eta_{\mathrm{p}}^{2}=.45$. The other significant main effect was that participants under high ETOD searched longer than those under low ETOD, $F(1,76)=157.16, p<.001$, $\eta_{\mathrm{p}}^{2}=.67$. We also identified a significant interaction, $F(1,76)=15.09, p<.001, \eta_{\mathrm{p}}^{2}=.17$. A simple effect analysis showed that there was a significant difference between FD and FI participants under high ETOD, $F(1$, $76)=23.13, p<.001$, whereas this difference was absent under low ETOD, $F(1,76)=2.73, p>.05$ (see Figure 1).

A subsequent 2 (field-cognitive style: $F I / F D) \times 2$ (ETOD: high/low) ANOVA on search depth revealed two significant main effects (Table 3). One was that FD participants acquired more information than FI participants, $F(1,76)=40.89, p<.001, \eta_{\mathrm{p}}^{2}=.35$. The other significant main effect was that participants under high ETOD acquired more information than those under low ETOD, $F(1,76)=104.38, \quad p<.001, \quad \eta_{\mathrm{p}}^{2}=.58$. Of particular interest was the significant interaction, $F(1,76)=4.96$, $p<.05, \eta_{\mathrm{p}}^{2}=.06$. Simple effect analysis showed that there was a significant difference between FD and FI participants under low ETOD, $F(1,76)=15.87, p<.001$, whereas only marginally significant difference was found under high ETOD, $F(1,76)=3.71, p=.058$ (see Figure 2).

Similarly, we conducted a 2 (field-cognitive style: FI/FD) $\times 2$ (ETOD: high/low) ANOVA on search pattern, which only revealed a single significant main effect (Table 4). Participants under high ETOD were more likely to use attribute-based processing, whereas participants with low ETOD were more likely to use alternative-based 


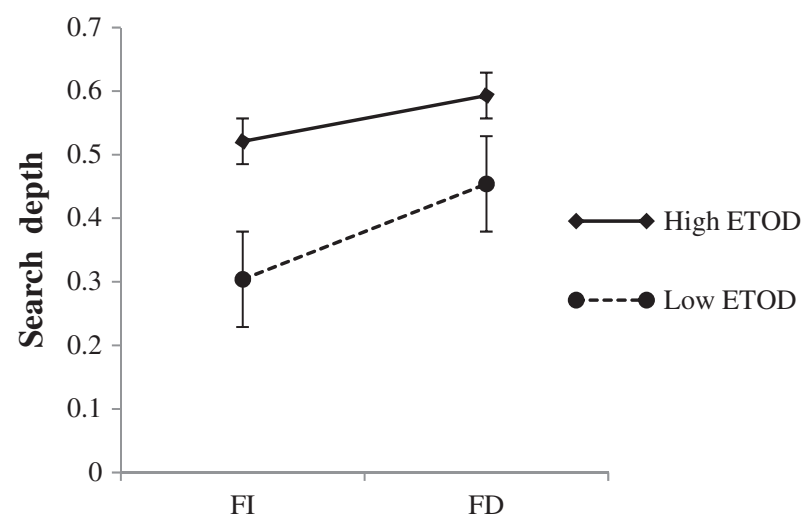

Figure 2. Interaction effect of field-cognitive style and ETOD on search depth.

TABLE 3

Search depth under high/low ETOD $(M \pm S D)$

\begin{tabular}{lcc}
\hline & High ETOD & Low ETOD \\
\hline FI & $0.52 \pm 0.07$ & $0.30 \pm 0.08$ \\
FD & $0.59 \pm 0.08$ & $0.45 \pm 0.08$ \\
\hline
\end{tabular}

TABLE 4

Search pattern under high/low ETOD $(M \pm S D)$

\begin{tabular}{lll}
\hline & High ETOD & Low ETOD \\
\hline FI & $-0.12 \pm 0.27$ & $0.10 \pm 0.27$ \\
FD & $-0.09 \pm 0.20$ & $0.13 \pm 0.22$ \\
\hline
\end{tabular}

processing, $F(1,76)=16.85, p<.001, \eta_{\mathrm{p}}^{2}=.18 \quad($ see Table 3). However, the main effect of field-cognitive style was not significant, $F(1,76)=0.30, p>.05, \eta_{\mathrm{p}}^{2}=.01$, nor was the interaction, $F(1,76)=0.001, p>.05, \eta_{\mathrm{p}}^{2}=.00$.

\section{DISCUSSION}

Our results demonstrate that participants experienced significant task-induced negative emotion when the level of ETOD switched from low to high, with increased search time and search depth. Furthermore, they were more likely to use attribute-based processing under high ETOD and alternative-based processing under low ETOD, a finding consistent with previous studies (Luce, 1998; Luce et al., 1997; Zhou, 2010).

Interestingly, these results can only be partially interpreted by the negative emotion hypothesis. The results seem generally consistent with one of the views of the hypothesis, namely that negative emotion subconsciously encourages or compels individuals to maximise their understanding of the decision-making environment. Specifically, we found that individuals were more likely to use alternative-based processing under low ETOD in order to increase accuracy. However, the switch of search pattern in our results under high ETOD, from alternative-based processing to attribute-based processing, cannot be interpreted under this framework. It may be the case that the negative emotion hypothesis, which is based on the effort-accuracy framework, only applies under low ETOD, or normal conditions (Luce et al., 2001).

The affect-as-information theory argues that emotion can serve as a source of information and hence influence decisions; emotional experiences provide adaptive advantages and play an important role in guiding and informing people's thought processes (Schwarz \& Clore, 2007). Similarly, negative emotion has been proposed as triggering a series of coping behaviours. The coping behaviour framework (Susan \& Lazarus, 1988) thus provides an alternative framework for interpreting the information processing that takes place during decision-making under ETOD. On the one hand, negative emotion may act as a signal that self-related outcomes are important, motivating greater problem-solving effort and processing accuracy on the part of the decision maker (Luce et al., 1997). Processing accuracy is generally associated with extensive processing. The perspective of the coping behaviour framework is thus consistent with our finding that search time and search depth increased along with the level of ETOD. On the other hand, emotion-focused coping may also make a difference by motivating the decision maker to avoid or reinterpret the subjective meaning of a situation. Such a strategy might result in more attribute-based processing, despite an increase in the overall extent of processing along with the increase in negative, task-related emotion. This scenario is also consistent with our results. Thus, the coping perspective suggests that negative emotion may have two simultaneous effects and two opposite motivations as ETOD increases. Emotion may serve as a signal regarding problem importance. At the same time, individuals may try to minimise the experience of negative emotion through avoidance (Luce et al., 1997). It follows that task-related emotion induced by trade-off difficulty might encourage decision makers to process both more extensively and using more attributed-processing under high ETOD.

Nevertheless, our observations regarding search pattern under low ETOD are not compatible with the coping behaviour framework. In light of this, we suggest a novel perspective, namely that the "negative emotion hypothesis" based on the effort-accuracy framework can be combined with the "coping behaviour framework" to more closely model information processing during decision-making under the condition of ETOD. That is to say, our results are best explained by the coping behaviour framework under high ETOD, and interpreted by the negative emotion hypothesis under low ETOD. For instance, an individual considering the decision in Table 1 may 
engage in an alternative-based processing pattern by combining his or her impressions of the traffic condition, security, household, location and property management offered by house 1 and then by other houses to form an overall assessment of all houses under low ETOD; they would then make a choice between the four houses whose attributes do not trigger obvious conflict. Conversely, under high ETOD, and with increasing negative emotion, people might engage in an attribute-based processing pattern by examining the traffic conditions of the four houses (the most important attribute to be considered); they might note that house 3 experiences the highest level of traffic, and make a decision with increased search time and search depth. While our results provide tentative evidence that both frameworks coexist and interact with each other under different levels of ETOD, this idea will need to be verified and elaborated on by future investigations.

In sum, our results show that ETOD affects information processing in decision-making, and suggest that this effect can be explained by the coping behaviour framework under high ETOD, and by the negative emotion hypothesis under low ETOD. In support of our hypotheses, we found that field-cognitive style interacted with ETOD to affect search time and search depth. However, contrary to our expectation, field-cognitive style did not moderate the impact of ETOD on search pattern. As the level of ETOD increased, the search pattern changed from alternative-based processing to attribute-based processing, regardless of FI or FD cognitive style. Research has shown that individuals use simple heuristic strategies to minimise negative emotion based on trade-off difficulty (Luce \& Payne, 2000). However, this goal of minimising negative emotion may be moderated by cognitive load. With a higher cognitive load, the relationship between self-relevant goals and negative emotion breaks down, reducing individuals' avoidance behaviour (Drolet \& Luce, 2004), and leading to a change in search pattern from attribute-based processing to alternative-based processing. This observation is of particular important to the present study, as individuals completed the decision task under a low cognitive load scenario, in which cognitive resources were not limited. Self-related goals and negative emotion were highly correlated, thus leading individuals to rely on attribute-based processing in order to reduce negative emotion. The drive to minimise negative emotion may explain why the search pattern was not affected by individual differences in cognitive style, and why it switched with increased level of ETOD.

\section{Limitations and future research}

Although valuable results have been obtained, several limitations of the present study should be noted. Firstly, although negative emotions were indeed generated during the evaluation process, the analysis was based on the assumption that these induced emotions were similar in valence across participants. A large number of studies have shown that emotions of different or even similar valence can affect decision-making in different ways. Accordingly, future research might focus on the types of specific emotion (such as anxiety and regret) that are triggered by ETOD, and their different impacts on decision-making. In addition, further work is needed to validate whether negative emotion provides an accurate description of the impact of ETOD on information processing. Another potentially worthwhile investigation would be to examine whether there are other individual variables that interact with ETOD to affect information processing. For example, Xu and Zhou (2010) found that participants with a "high need for cognition" tended to use analytical strategies, whereas participants with a "low need for cognition" tended to use heuristic strategies. The potential interaction between need for cognition and ETOD on information search in decision-making might provide an interesting direction for further research.

\section{CONCLUSION}

In sum, ETOD is an important variable that influences information processing during decision-making, with significant negative emotion likely to be evoked as ETOD increases. We found that field-cognitive style interacted with ETOD to influence search time and search depth. Individuals consistently resorted to attribute-based processing under high levels of ETOD and alternative-based processing under low levels of ETOD. In addition to the traditional negative emotion hypothesis, we have proposed that the coping behaviour framework can also be integrated into the toolkit to better explain and model these observations.

\author{
Manuscript received January 2016 \\ Revised manuscript accepted November 2016 \\ First published online December 2016
}

\section{REFERENCES}

Bandyopadhyay, D., Srinivasan, N., \& Pammi, V. S. C. (2013). Dissociable effects of task-irrelevant emotional information on decision making under risk. Neuroscience of Decision Making, 1, 1-8.

Baumgartner, H., Pieters, R., \& Bagozzi, R. P. (2008). Future-oriented emotions: conceptualization and behavioral effects. European Journal of Social Psychology, 38(4), 685-696.

Chen, Y. (2011). Experiment study of advice taking under multi-advices situation. Master's thesis, Jinan University.

Damasio, A. R. (1995). Review: Toward a neurobiology of emotion and feeling: operational concepts and hypotheses. The Neuroscientist, 1, 19-25. 
Drolet, A., \& Luce, M. F. (2004). The rationalizing effects of cognitive load on emotion-based trade-off avoidance. Journal of Consumer Research, 31(1), 63-77.

Lazarus, R. S. (1991). Progress on a cognitive-motivationalrelational theory of emotion. American Psychologist, 46(8), 819-883.

Lerner, J. S., Li, Y., Valdesolo, P., \& Kassam, K. S. (2015). Emotion and decision making. Annual Review of Psychology, 66(1), 799-823.

Lewinsohn, S., \& Mano, H. (1993). Multi-attribute choice and affect: The influence of naturally occurring and manipulated moods on choice processes. Journal of Behavioral Decision Making, 6(1), 33-51.

Li, X. M., \& Fu, X. L. (2004). Decision behavior in emotional trade-off difficulties. Advances in Psychological Science, 12(6), 801-808.

Li, X. M., Huang, R., \& Zhou, X. (2015). The effects of accidental emotions on decision making. Advances in Psychological Science, 23(6), 919-925.

Luce, M. F. (1998). Choosing to avoid: coping with negatively emotion-laden consumer decisions. Journal of Consumer Research, 24(4), 409-433.

Luce, M. F., Bettman, J. R., \& Payne, J. W. (1997). Choice processing in emotionally difficult decisions. Journal of Experimental Psychology: Learning, Memory, and Cognition, 23(2), 384-405.

Luce, M. F., Bettman, J. R., \& Payne, J. W. (2001). Emotional decisions: Trade-off difficulty and coping in consumer choice. Monographs of the Journal of Consumer Research, $1,1-209$.

Luce, M. F., Payne, J. W., \& Bettman, J. R. (2000). Coping with unfavorable attribute values in choice. Organizational Behavior and Human Decision Processes, 81(2), 274-299.

Luo, X. Y. (2011). The research of cognitive style, construal level and emotion affecting impulse behavior. Master's thesis, Northwest Normal University.

Mawad, F., Trías, M., Giménez, A., Maiche, A., \& Ares, G. (2015). Influence of cognitive style on information processing and selection of yogurt labels: Insights from an eye-tracking study. Food Research International, 74, 1-9.

Palmer, A., \& Koenig-Lewis, N. (2014). The effects of anticipatory emotions on service satisfaction and behavioral intention. Journal of Services Marketing, 28, 437-451.

Payne, J. W. (1976). Task complexity and contingent processing in decision making: An information search and protocol analysis. Organizational Behavior and Human Performance, 16(76), 366-387.

Payne, J. W., Bettman, J. R., \& Eric, J. (1993). The adaptive decision maker. Cambridge: Cambridge University.

Peng, H. (2008). The effects of time pressure to decision behavior in emotional trade-off difficulty. Master's thesis, Hunan Normal University.

Qiu, F. D., \& Wu, M. Z. (2005). An experimental research on the influence of cognitive styles and negative emotions on tourism decision-making. Psychological Science, 28(5), $1112-1114$

Schwarz, N., \& Clore, G. L. (2007). Feelings and phenomenal experiences. In A. Kruglanski \& E. T. Higgins (Eds.), Social psychology: Handbook of principles (2nd ed., pp. 385-407). New York, NY: Guilford.
Simon, H. A. (1983). Reason in human affairs. Stanford, CA: Stanford University Press.

Susan, F., \& Lazarus, R. S. (1988). Coping as a mediator of emotion. Journal of Personality and Social Psychology, 54(3), 466-475.

Watson, D., Clark, L. A., \& Tellegen, A. (1988). Development and validation of brief measures of positive and negative affect: the PANAS scales. Journal of Personality and Social Psychology, 54(6), 1063-1070.

Witkin, H. A. (1964). Origins of cognitive style. In C. Sheerer (Ed.), Cognition: Theory, research, promise. New York: Harper \& Row.

Xie, J., \& Zhang, H. (1988). Cognitive style: Experimental study on a personality dimension. Beijing: Beijing Normal University Press.

Xu, J., \& Zhou, N. (2010). The effects of need for cognition on the dispositional differences of individuals' information processing. Advances in Psychological Science, 4(4), 685-690.

Zhang, W. D., Diao, J., \& Constance, J. S. (2004). The cross-cultural measurement of positive and negative affect examining the dimensionality of PANAS. Psychological Science, 27(1), 77-79.

Zhou, W. (2010). The effects of time pressure and emotional trade-off difficulty to choice deferral. Master's thesis, East China Normal University.

\section{APPENDIX}

The following materials for emotional trade-off difficulty were developed based on analysis of participants' beliefs regarding the importance and loss aversion associated with each relevant attribute.

Data were collected for three different measures of importance.

1 Pricing improvement. Participants were asked to define an RMB $¥$ value equivalent to changing a given attribute of a house from "average" to " $20 \%$ improvement (above average)".

2 Swing improvement. Participants were asked to mentally construct a house in the worst possible condition for every attribute. They were then asked to indicate which attribute they would most like to change from its worst to its best value, assigning that attribute a value of 100. Participants also rated the urgency of changing the condition of each of the remaining 14 attributes from worst to best by assigning values to these attributes relative to the initial 100 value.

3 Ratio weights. Participants were given a list of all 15 attributes of a house and asked to first assign a value of 100 to the attribute that was most important to them. Then, participants were asked to assign values reflecting the importance of the remaining attributes relative to 100 .

Data were also collected for three measures of loss aversion. 
TABLE A1

Average importance and loss aversion measures for high and low trade-off difficulty attributes

\begin{tabular}{|c|c|c|c|c|c|c|}
\hline & \multicolumn{3}{|c|}{ Loss aversion measures } & \multicolumn{3}{|c|}{ Importance measures } \\
\hline & Pricing decrement & Swing decrement & Risk attitude & Pricing improvement & Swing improvement & Ratio weights \\
\hline House type & 5.19 & 5.17 & 5.07 & 5.02 & 4.79 & 4.90 \\
\hline Traffic condition & 5.61 & 6.01 & 6.07 & 5.03 & 5.06 & 5.15 \\
\hline Space interval & 4.30 & 3.95 & 3.86 & 5.01 & 5.02 & 4.89 \\
\hline Parking lot & 3.23 & 3.23 & 3.14 & 3.27 & 3.22 & 3.37 \\
\hline Light condition & 3.43 & 3.45 & 3.36 & 3.08 & 3.07 & 3.16 \\
\hline Ventilation & 3.68 & 3.61 & 3.49 & 3.93 & 3.84 & 3.81 \\
\hline Architectural style & 3.40 & 3.70 & 3.89 & 3.15 & 3.19 & 3.23 \\
\hline Size of community & 3.49 & 3.46 & 3.52 & 3.68 & 3.67 & 3.53 \\
\hline Residential scale & 3.80 & 3.66 & 3.61 & 3.87 & 4.08 & 3.73 \\
\hline Educational facilities & 3.02 & 3.19 & 3.11 & 3.01 & 2.98 & 3.24 \\
\hline Landscape & 3.95 & 3.79 & 3.86 & 4.02 & 4.03 & 3.96 \\
\hline Property management & 4.71 & 4.53 & 4.57 & 4.70 & 4.81 & 4.82 \\
\hline Location & 4.88 & 4.96 & 4.95 & 4.97 & 4.91 & 4.91 \\
\hline Security & 4.02 & 4.09 & 4.12 & 3.59 & 3.66 & 3.61 \\
\hline Entertainment & 3.43 & 4.09 & 4.12 & 3.59 & 3.66 & 3.61 \\
\hline
\end{tabular}

Note: Information in this table is cited from Zhou (2010).

1 Pricing decrement. Participants were asked to imagine that they possessed a house with average values on all attributes, and to report the amount in RMB they would request in return for accepting a $20 \%$ decrease on each attribute.

2 Swing decrement. This measure asked participants to imagine that they possessed a house with the best value for all attributes, and to rate their reluctance of reducing the best value to the worst value for each attribute. The attribute that they were most reluctant to change was given a value of 100, and attributes for which they were less reluctant to accept the quality reduction were given lower values.

3 Risk attitude. Participants were asked to make a choice between a sure thing and a gamble involving a 50/50 chance of a gain or a loss. They completed questions with the following form:

I am indifferent between House A with an average level in attribute $\alpha$ and House $B$ with a

- $50 \%$ chance of a $20 \%$ decrease in attribute $\alpha$; and

- $50 \%$ chance of an $\underline{n} \%$ increase in attribute $\alpha$.

Participants were asked to indicate the value " $n$ " reflecting the percentage of increase in quality of attribute $\alpha$ (with 50/50 odds) that they would seek to balance out the $50 \%$ chance of a $20 \%$ decrease in that attribute. Larger values for this response reflect greater loss aversion.

Next, we established the trade-off difficulty by manipulating the attributes. For the high trade-off difficulty attribute sets, we held the three importance measures constant and allowed the loss aversion measures to fluctuate. For low trade-off difficulty attribute sets, we let all the six measures fluctuate.

The pretest data were standardised by participant and then used to identify pairs of attributes with the following qualities: (a) the attributes in the pair did not significantly differ from one another in terms of importance, and (b) the attributes in the pair did significantly differ from each other in terms of loss aversion.

In China, college students would not usually have considered these specific issues before participating in the experiment. As a result, emotional trade-off difficulty was relatively less affected by individual differences and demographics, such as family background, income, age, occupation, and lifestyle. The results also showed that participants with high emotional trade-off difficulty experienced considerably more negative emotion during the weighing process (see Zhou, 2010). 